\title{
Identification of crucial genes associated with rat traumatic spinal cord injury
}

\author{
ZIBIN YANG, QIAO LV, ZHENGXIANG WANG, XILIANG DONG, RONGXIN YANG and WEI ZHAO \\ Department of Spinal Surgery, The People's Hospital of Dali Prefecture, Dali, Yunnan 671000, P.R. China
}

Received December 13, 2015; Accepted December 9, 2016

DOI: $10.3892 / \mathrm{mmr} .2017 .6267$

\begin{abstract}
The aim of the present study was to investigate the key genes associated with traumatic spinal cord injuries (TSCI). The dataset GSE52763 was downloaded from the Gene Expression Omnibus, for which lumbar spinal cord samples were obtained from rats at 1 and 3 weeks following contusive spinal cord injury and 1 week subsequent to a sham laminectomy, and used to identify differentially expressed genes (DEGs). Functional enrichment analysis, co-expression analysis and transcription factor (TF) identification were performed for DEGs common to the 1 and 3 week injury samples. In total, 234 upregulated and 51 downregulated DEGs were common to the 1 and 3 week injury samples. The upregulated DEGs were significantly enriched in Gene Ontology terms concerning immunity (e.g. Itgal and Ccl2) and certain pathways, including natural killer cell mediated cytotoxicity [e.g. Ras-related C3 botulinum toxin substrate 2 (Rac2) and TYRO protein tyrosine kinase binding protein (Tyrobp)]. The downregulated DEGs were highly enriched in female gonad development [e.g. progesterone receptor $(P g r)]$, and the steroid biosynthesis pathway. A total of 139 genes had co-expression associations and the majority of them were upregulated genes. The upregulated co-expressed genes were predominantly enriched in biological regulation, including TGFB induced factor homeobox 1 (Tgif1) and Rac2. The downregulated co-expressed genes were enriched in anatomical structure development (e.g. Dnm3). A total of 92 co-expressed genes composed the protein-protein interaction network. Additionally, 9 TFs (e.g. Pgr and Tgifl) were identified from the DEGs. It was hypothesized that the genes including Tgif1, Rac2, Tyrobp, and Pgr may be closely associated with TSCI.
\end{abstract}

Correspondence to: Professor Wei Zhao, Department of Spinal Surgery, The People's Hospital of Dali Prefecture, 35 Renmin Road, Dali, Yunnan 671000, P.R. China

E-mail: zhaowieieii@hotmail.com

Key words: co-expression, differentially expressed gene, network, traumatic spinal cord injury, transcription factor

\section{Introduction}

Patients with traumatic spinal cord injuries (TSCI) endure low health-associated quality of life and high healthcare costs. They also have a higher mortality rate compared with the general population. In 2012, the estimated incidence of acute spinal cord injury in the United States was 54 cases per 1 million (1). The biological processes of TSCI involve a diverse group of cells and molecules from the nervous, immune and vascular systems. For instance, connexin 43 functions as a mediator of central nervous system inflammation and chronic pain following spinal cord injury (2), altered expression of E2F-associated phosphoprotein regulates reactive astrogliosis and neuronal apoptosis (3) and ginsenoside Rb1 upregulates the expression of Bcl-xL and vascular endothelial growth factor at 7 days after spinal cord injury (4). Investigation of gene changes has contributed to the understanding of the molecular mechanisms of TSCI.

Gene expression profiling by microarray has been used to uncover molecular variations in spinal cord repair and degeneration (5-7). In 2014, using microarray analysis, Shin et al (8) identified that numerous inflammation-associated genes were upregulated in the lumbar spinal cord at 1 and 3 weeks after traumatic injury, and locomotor function was improved in part by treadmill locomotor training (TMT). However, the molecular mechanisms of TSCI remain to be elucidated and regulatory factors associated with TSCI, including transcription factors (TFs), have not been investigated to the best of the authors' knowledge.

The present study used the microarray data obtained by Shin et al (8) and screened differentially expressed genes (DEGs) common to the 1 and 3 week injury samples, and then analyzed the functions and interactions of DEGs. Additionally, TFs in DEGs were identified to reveal the regulatory associations of DEGs. These results may provide novel information to aid the understanding of the molecular mechanisms of TSCI.

\section{Materials and methods}

Affymetrix microarray data. The raw gene expression profile data GSE52763 (8) were obtained from the public database Gene Expression Omnibus (GEO; http://www.ncbi.nlm. nih.gov/geo/), which is based on the platform of GPL1355 (Rat230_2) Affymetrix Rat Genome 230 2.0 Array. The 
dataset contained eight rat lumbar spinal cord samples obtained from rats $1(n=4)$ and $3(n=4)$ weeks following contusive spinal cord injury at the T9 level (designated as 1 week injury and 3 weeks injury samples), three lumbar spinal cord samples obtained from rats 1 week following sham laminectomy (designated as sham samples), four lumbar spinal cord samples obtained at 3 weeks following contusive spinal cord injury with treadmill training (designated as 3 weeks injury + TMT samples), and three lumbar spinal cord samples from rats which underwent a sham laminectomy followed by 2 weeks of treadmill training (designated as sham + TMT samples). All of the samples were taken from adult (8 weeks) female Sprague-Dawley rats (250-300 g). Only 1 week injury, 3 weeks injury and sham samples were used for analysis in the present study.

CEL files were downloaded and the gene expression data of all samples were preprocessed through background correction, quantile normalization and probe summarization using the Robust Microarray Analysis algorithm of the affy package of Bioconductor (http://www.bioconductor.org/packages/ release/bioc/html/) (9).

DEGs screening. The linear models for the microarray data package of Bioconductor (10) was used to identify DEGs between 1 week and 3 weeks injury samples and sham samples. The P-value for each gene was calculated by $t$-test and only the genes with P-value $<0.05$ and fold change $\geq 1.5$ were selected as DEGs. Subsequently, the DEGs common to the 1 and 3 week injury samples were screened for subsequent analyses.

Enrichment analysis. Gene Ontology (GO) functional and Kyoto Encyclopedia of Genes and Genomes (KEGG) pathway enrichment analyses for DEGs were conducted using the Database for Annotation, Visualization and Integrated Discovery (http://david.abcc.ncifcrf.gov) database, which provides a set of functional annotation tools to aid investigators in comprehending the biological importance underlying numerous genes (11). $\mathrm{P}<0.05$ and gene count $\geq 2$ were set as the cut-off criteria.

Co-expression analysis. The Pearson correlation coefficient was calculated to analyze the co-expression associations between DEGs (11). The co-expression pairs with a Pearson correlation coefficient $>0.9$ were screened out, and the co-expression network was visualized using Cytoscape [http://cytoscape.org; (12)].

Subsequently, GO functional enrichment analysis in biological process was performed using the plug-in Bingo (13) in Cytoscape. $\mathrm{P}<0.05$ was set as the cut-off criterion. Additionally, the Search Tool for the Retrieval of Interacting Genes/Proteins (http://string-db.org) database was used to analyze the protein-protein interactions (PPIs) of co-expressed genes, and the PPI network was visualized by Cytoscape.

Identification of TFs from DEGs. TFs in the DEGs common to the 1 and 3 week injury samples were identified using the Animal Transcription Factor Database [AnimalTFDB; http:// www.bioguo.org/AnimalTFDB/species_index.php; (14)].
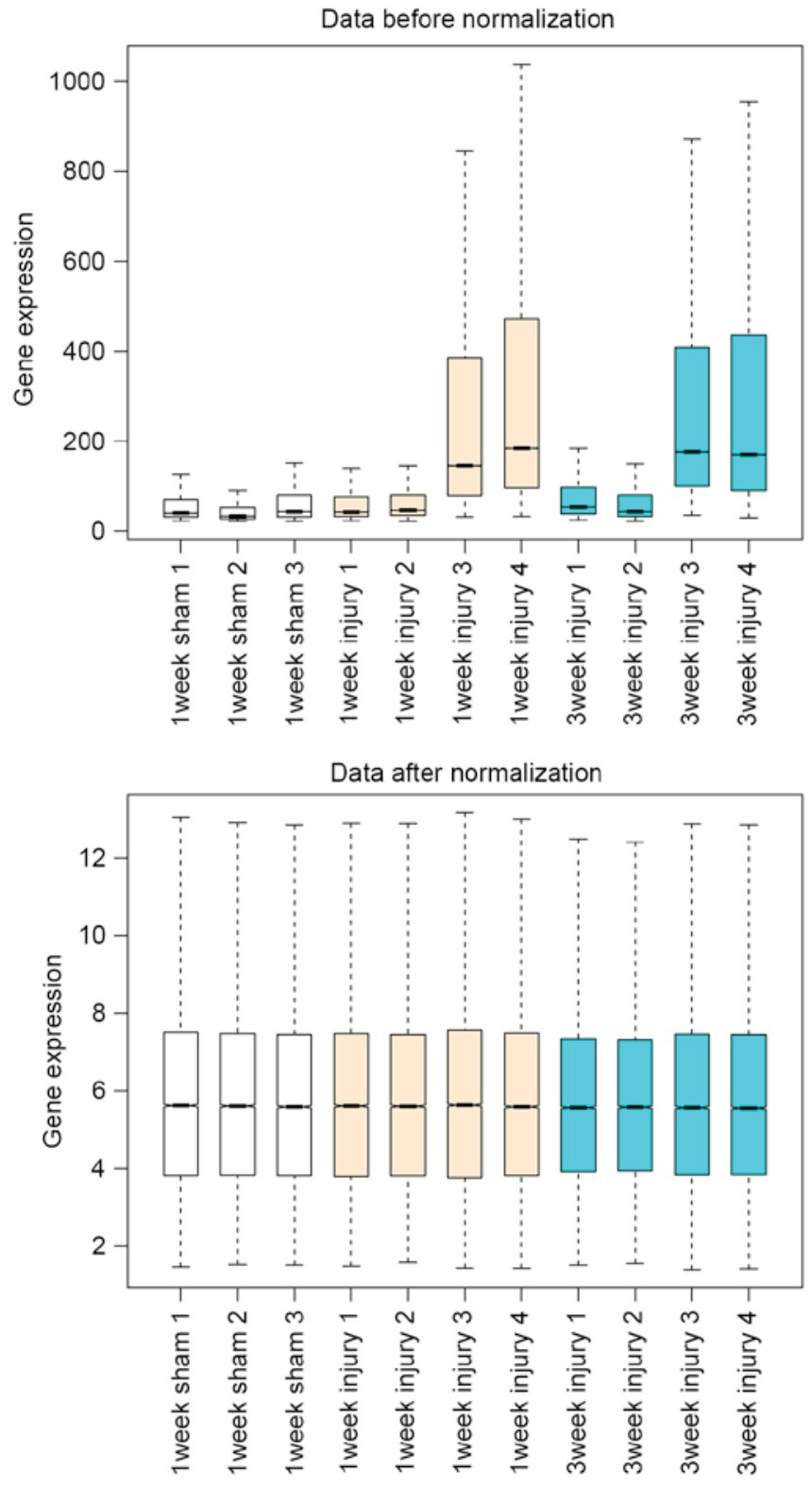

Figure 1. The boxplots for microarray data prior and subsequent to normalization. The abscissa displays the samples and the ordinate represents gene expression. ' 1 week sham' represents lumbar spinal cord samples obtained from rats 1 week following a sham laminectomy, three repeats; ' 1 week injury' and ' 3 week injury' represents lumbar spinal cord samples obtained from rats 1 and 3 weeks following contusive spinal cord injury at the T9 level (four repeats for each treatment).

\section{Results}

Identification of DEGs. Based on the normalization of the microarray data, the boxplot of preprocessed data displayed that medians of each sample data were almost on a line, indicating that the data after preprocessing met the standard for further analyses (Fig. 1).

In total, 322 upregulated and 78 downregulated DEGs were screened between 1 week injury and sham samples, in addition to 354 upregulated and 285 downregulated ones between 3 week injury and sham samples. Among them, 234 upregulated and 51 downregulated DEGs were common to the 1 and 3 week injury samples. The hierarchical cluster analysis of the data demonstrated that the DEGs can be used to 


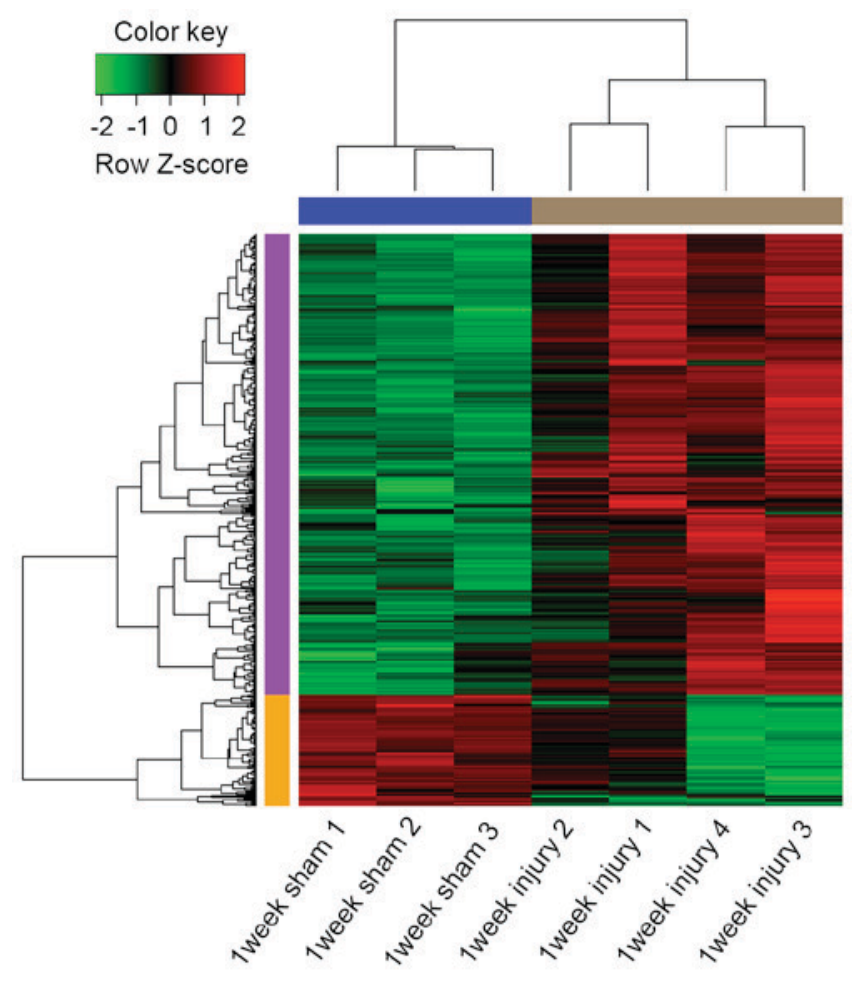

GO functional and KEGG pathway enrichment analyses. To identify the functions of DEGs common to the 1 and 3 week injury samples, GO functional and KEGG pathway enrichment analyses were performed. According to GO functional enrichment analysis, the upregulated DEGs were significantly enriched in several GO terms concerning immunity, including immune response [e.g. integrin subunit $\alpha \mathrm{L}$ (Itgal), similar to guanylate binding protein family, member 6 and $\mathrm{C}-\mathrm{C}$ motif chemokine ligand $2(\mathrm{Ccl} 2)$ ], defence response (e.g. TNF $\alpha$ induced protein 8 like 2, apolipoprotein B MRNA editing enzyme catalytic subunit 1 and $C c l 2$ ) and cell activation (e.g. exonuclease 1, Intercellular Adhesion Molecule 1 and pleckstrin; Table I). The downregulated DEGs were highly enriched in female gonad development [e.g. Pgr, vascular endothelial growth factor A (Vegfa) and BCL2 like 1], neuron projection [e.g. ATPase plasma membrane $\mathrm{Ca}^{2+}$ transporting 2 , dynamin $3(\mathrm{Dnm} 3)$ and glutamate metabotropic receptor 7 $(\mathrm{Grm} 7)]$ and gated channel activity [e.g. $\gamma$-aminobutyric acid type A receptor $\alpha 3$ subunit (Gabra3), Grm7 and calcium voltage-gated channel auxiliary subunit $\beta 4$; Table II].

Meanwhile, a set of upregulated DEGs were markedly enriched in certain pathways, including natural killer cell mediated cytotoxicity [e.g. Ras-related $\mathrm{C} 3$ botulinum toxin substrate 2 (Rac2) and TYRO protein tyrosine kinase binding protein (Tyrobp)] and the B cell receptor signaling pathway [e.g. fc fragment of IgG receptor IIb $(F \operatorname{cg} r 2 B)$ and Rac2). Several downregulated DEGs were significantly enriched in the pathways of steroid biosynthesis (cytochrome P450 family 51 , transmembrane 7 superfamily member 2 and lanosterol synthase) and neuroactive ligand-receptor interaction (e.g. Gabra3 and Grm7; Table III).

Analysis of co-expressed genes. Gene co-expression analysis is a powerful method to predict the function of genes and/or to identify genes that are functionally associated with query genes. Based on the cut-off criterion of Pearson correlation coefficient $>0.9,1894$ co-expression pairs in 139 DEGs were obtained (Fig. 3). Notably, the majority of co-expressed genes were upregulated DEGs.

According to GO functional enrichment analysis, the upregulated co-expressed genes [e.g. Rac2, fc fragment of IgG receptor Ia and including TGFB induced factor homeobox 1 (Tgifl)] were significantly enriched in a series of GO terms, including biological regulation and response to stimulus. The downregulated co-expressed genes were markedly enriched in certain GO terms, including anatomical structure development (e.g. Dnm3 and Vegfa) and intracellular signal transduction (e.g. mitogen-activated protein kinase kinase kinase kinase 5 and $\operatorname{Grm} 7$ ) (Table IV).

The PPI network was composed of 92 co-expressed genes

Figure 2. The cluster heat maps for the differentially expressed gene between the sham samples and the 1 and 3 week injury samples. Each row represents a single gene and each column represents a spinal cord sample. The gradual color change from red to green represents the changing process from upregulation to downregulation. ' 1 week sham' represents lumbar spinal cord samples obtained from rats 1 week following a sham laminectomy, three repeats; ' 1 week injury' and '3 week injury' represents lumbar spinal cord samples obtained from rats 1 and 3 weeks following contusive spinal cord injury at the T9 level (four repeats for each treatment).

accurately distinguish 1 and 3 week injury samples from sham samples (Fig. 2). (83 upregulated and 9 downregulated) and 351 interactions. The connectivity degree of six genes was more than 20 and they were Tyrobp (degree $=35)$, CD68 molecule $($ Cd68; degree $=34)$, Rac2 (degree=29), integrin subunit $\beta 2$; (degree=28), CD53 molecule (degree=25), C-type lectin domain family 4 member A (degree $=22)$. Tyrobp interacted with multiple genes, including Cd68 and Rac2 (Fig. 4).

Analysis of TFs. Based on the AnimalTFDB database, a total of 9 TFs were identified from the DEGs common to the 1 


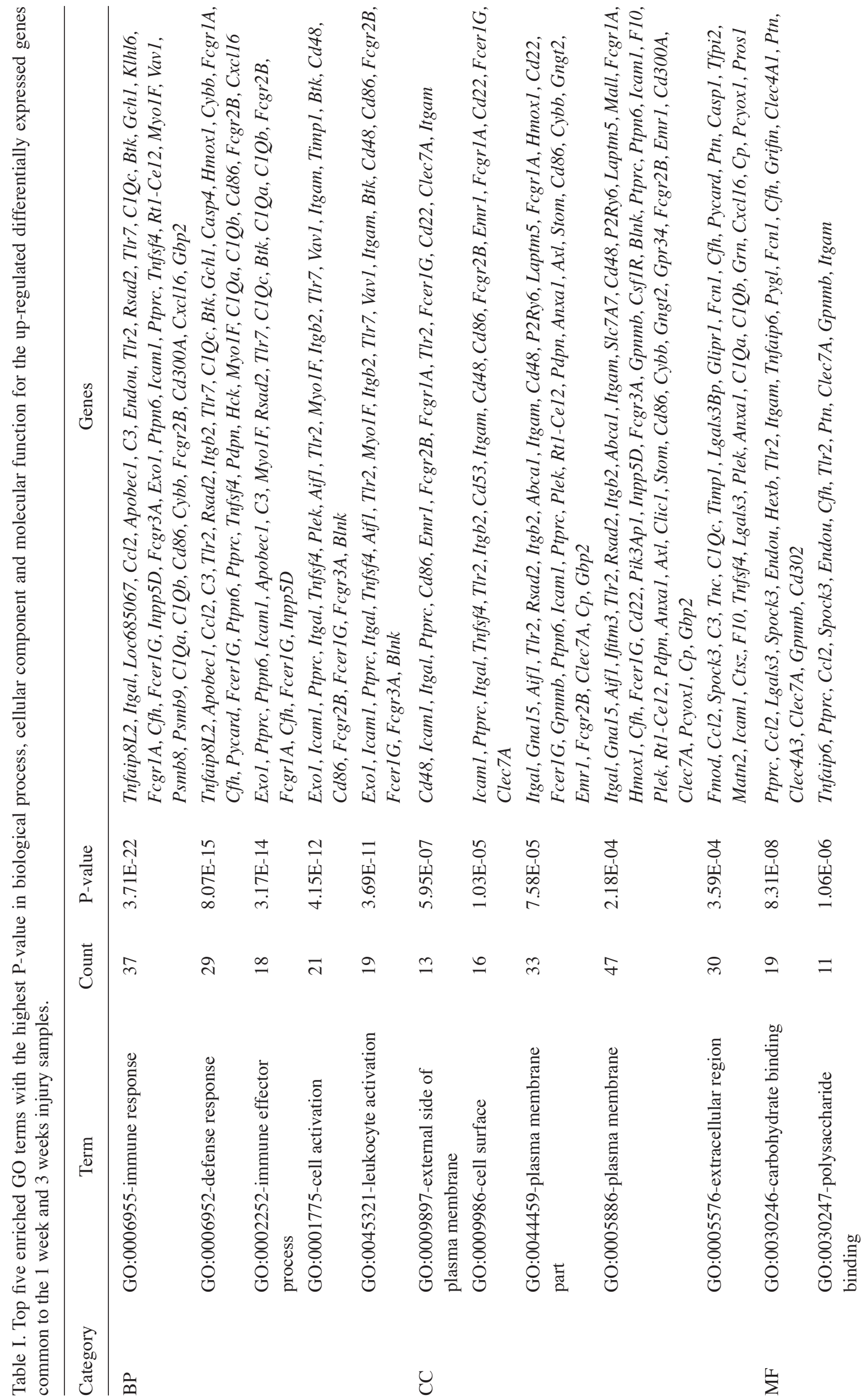




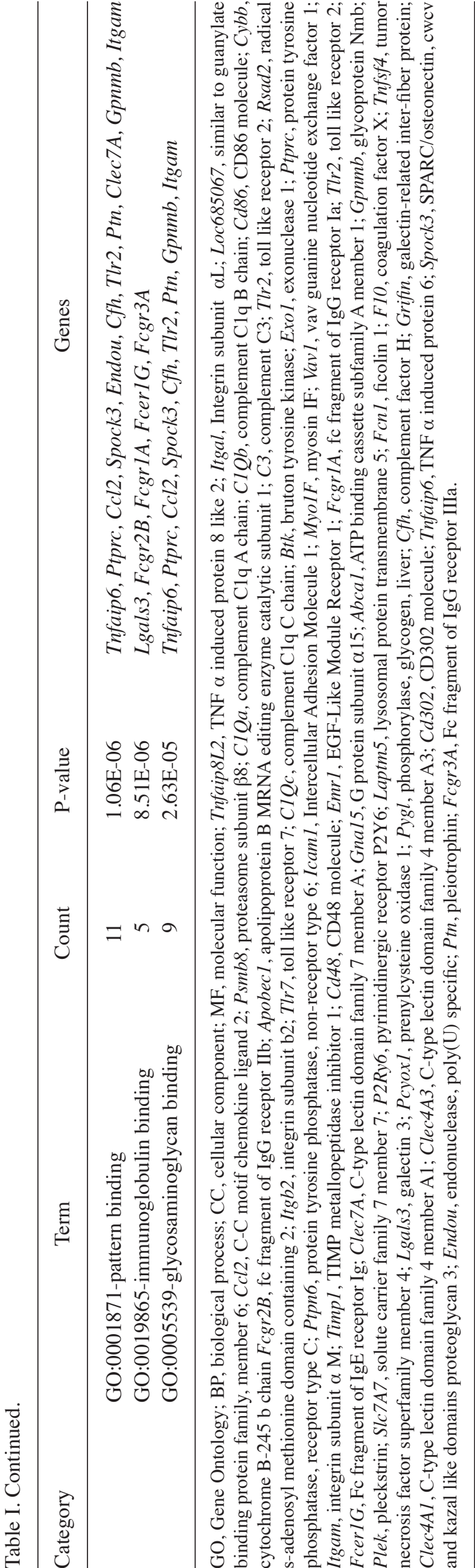

and 3 week injury samples, including cold shock domain containing $\mathrm{C} 2, \mathrm{Pgr}$, zinc finger and BTB domain containing 7B, SRY-box 18, activating TF 3 (Atf3), MAF BZIP TF B (Mafb), Tgif1, Fli-1 proto-oncogene, ETS TF (Flil) and T-box 4 (Tbx4). Among them, Atf3, Mafb, Tbx4, Tgif1 and Fli1 were all upregulated in 1 and 3 week injury samples, compared with sham samples, while the others were downregulated (Fig. 5).

\section{Discussion}

In the present study, 234 upregulated and 51 downregulated DEGs were common to the 1 week and 3 week injury samples, compared with the sham samples. Among them, 139 genes had co-expression associations and the majority of them were upregulated genes. The upregulated co-expressed genes were predominantly enriched in several GO terms of biological regulation, including Tgifl and Rac2.

Tgifl was identified as a TF in the present study. It belongs to the three-amino acid loop extension superclass of atypical homeodomains (15). Studies (16-18) have showed that Tgifl exerts crucial functions in the nervous system. Additionally, a previous study (19) identified TGIF1 as a novel regulator of macrophage activation in immune response. In the present study, Tgifl had a co-expression associations with Rac2 and Tyrobp, the two of which had a higher degree in the PPI network. Rac2 encodes a member of the Ras superfamily of small guanosine triphosphate (GTP)-metabolizing proteins, and it modulates diverse processes, including secretion, cell polarization and phagocytosis (20). In the present study, Rac2 was identified to be significantly enriched in several pathways, including natural killer cell-mediated cytotoxicity. Natural killer cells participate in immune processes after spinal cord injury (21) and there is evidence that suppression of Rac activity in the injured spinal cord enhances cell survival (22). It has been demonstrated that the expression of Rac2 is activated in inflammatory responses (23). Furthermore, Ras GTPases exert critical functions in multiple procedures during axonogenesis in injured spinal cords (24). Tyrobp is a transmembrane signaling polypeptide which has an immunoreceptor tyrosine-based activation motif and it serves a role in signal transduction, brain myelination, and inflammation $(25,26)$. In the current study, Tyrobp was enriched in natural killer cell mediated cytotoxicity, interacted with Rac2 in the PPI network and co-expressed with Tgif1. Together, Tgif1, Rac2 and Tyrobp may play pivotal roles in TSCI.

Among the downregulated genes, Pgr, identified as a $\mathrm{TF}$, was highly enriched in female gonad development and the ovulation cycle process. In axonal regeneration, gonadal steroids function as promoting factors (27). Estrogens have direct neuroprotective effects, including modification of humoral immune responses, and gestagens can prevent neuronal death and promote the growth of nervous cells and the formation of new synapses (28). A previous study (29) confirmed that progesterone provides neuroprotection to the injured central and peripheral nervous system in the injured spinal cord. Therefore, Pgr may serve a key role in the regulation of nervous regeneration in spinal cord injuries.

In conclusion, 234 upregulated and 51 downregulated DEGs were differentially expressed in 1 and 3 week injury samples. Among them, the upregulated genes Rac2 and 


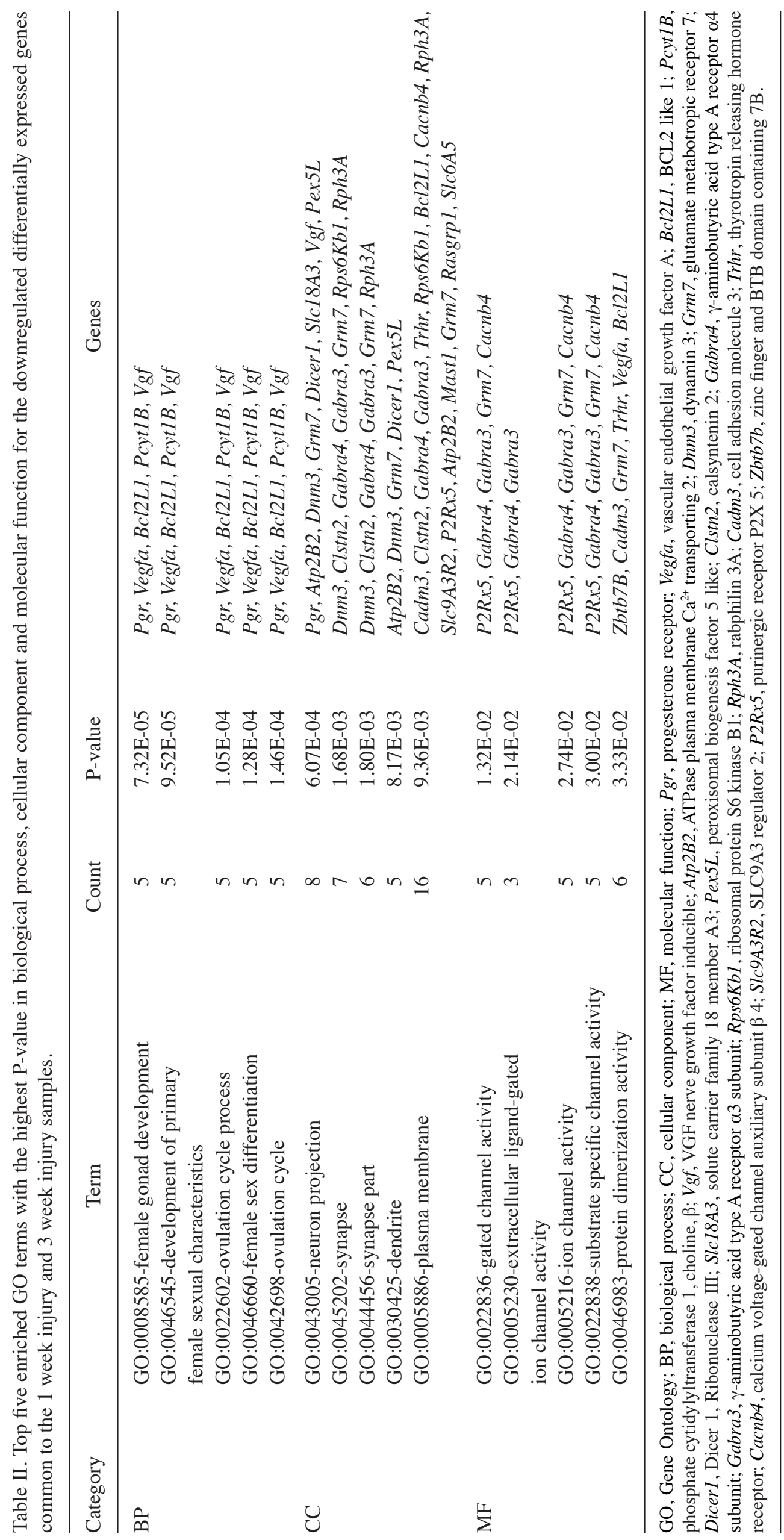




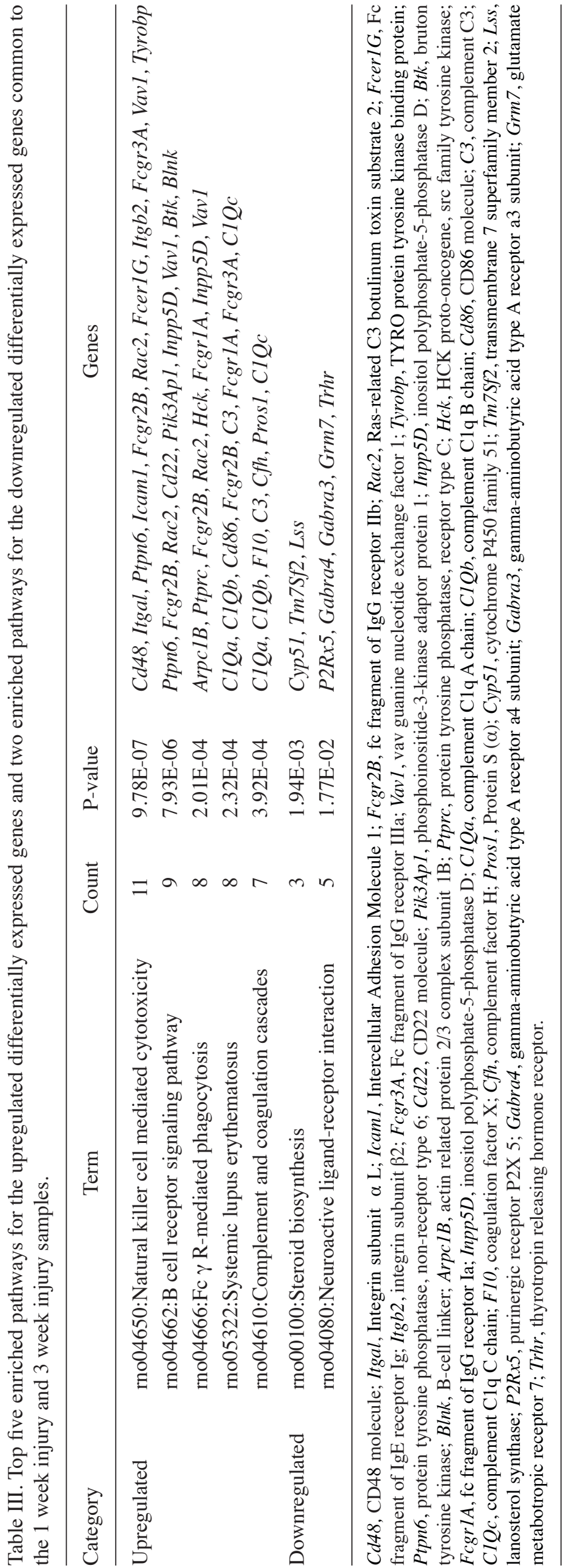




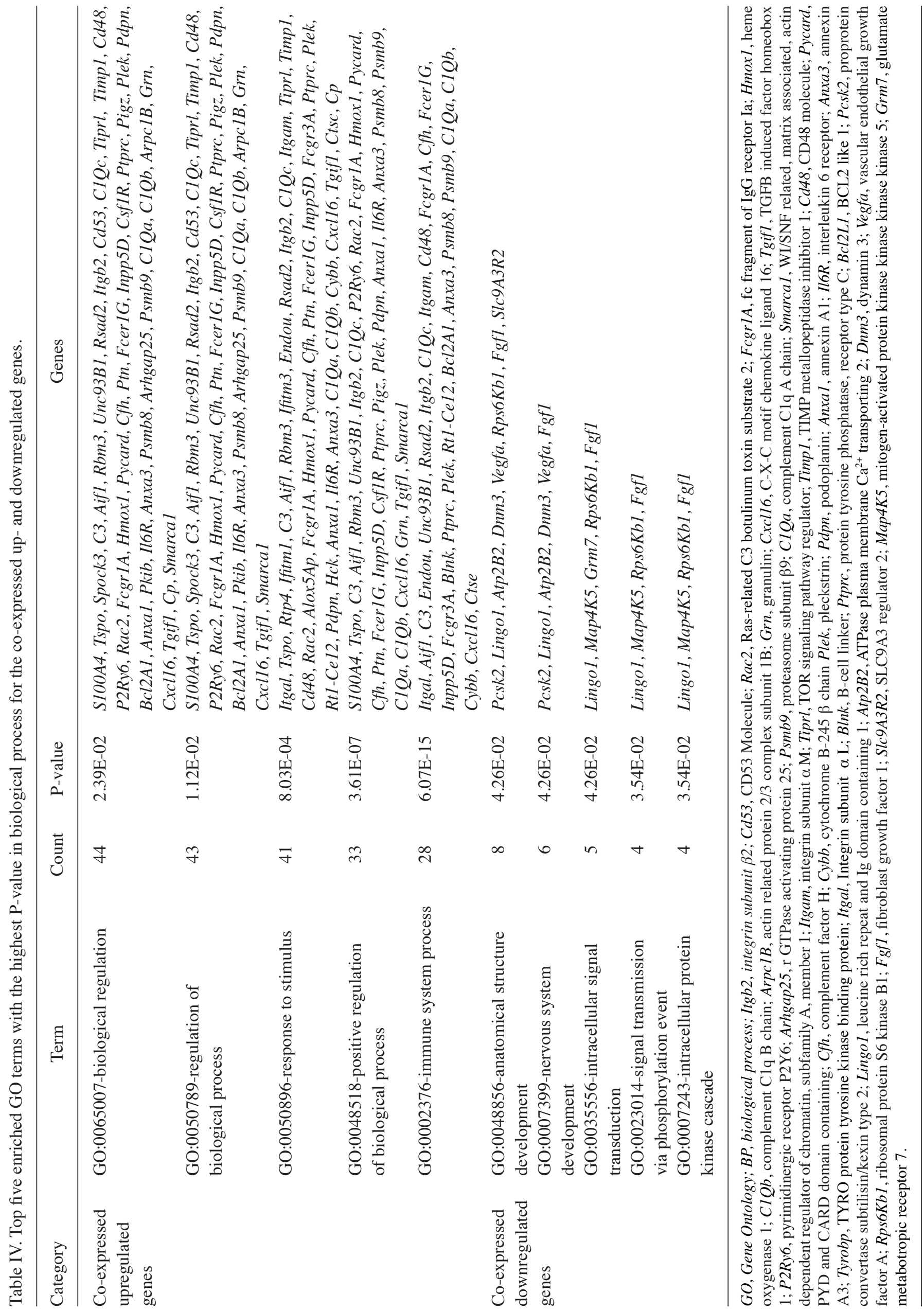




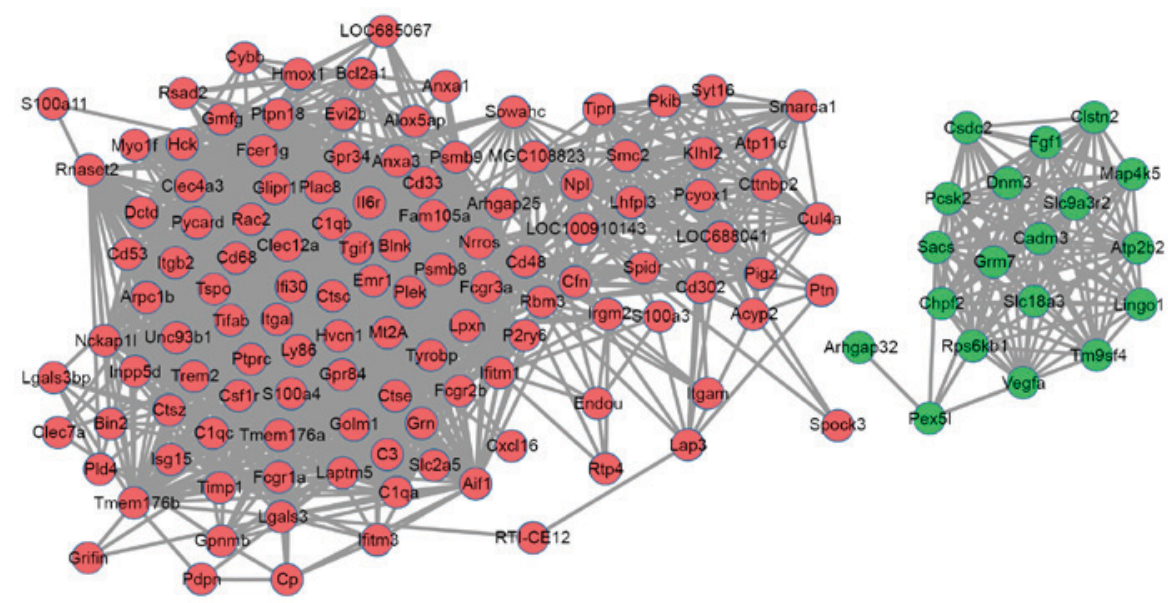

Figure 3. The co-expression network of the differentially expressed genes common to the 1 week and 3 week injury samples. The red nodes represent upregulated genes; and the green nodes represent downregulated genes. A line between two nodes indicates that there is a co-expression association between these two nodes.

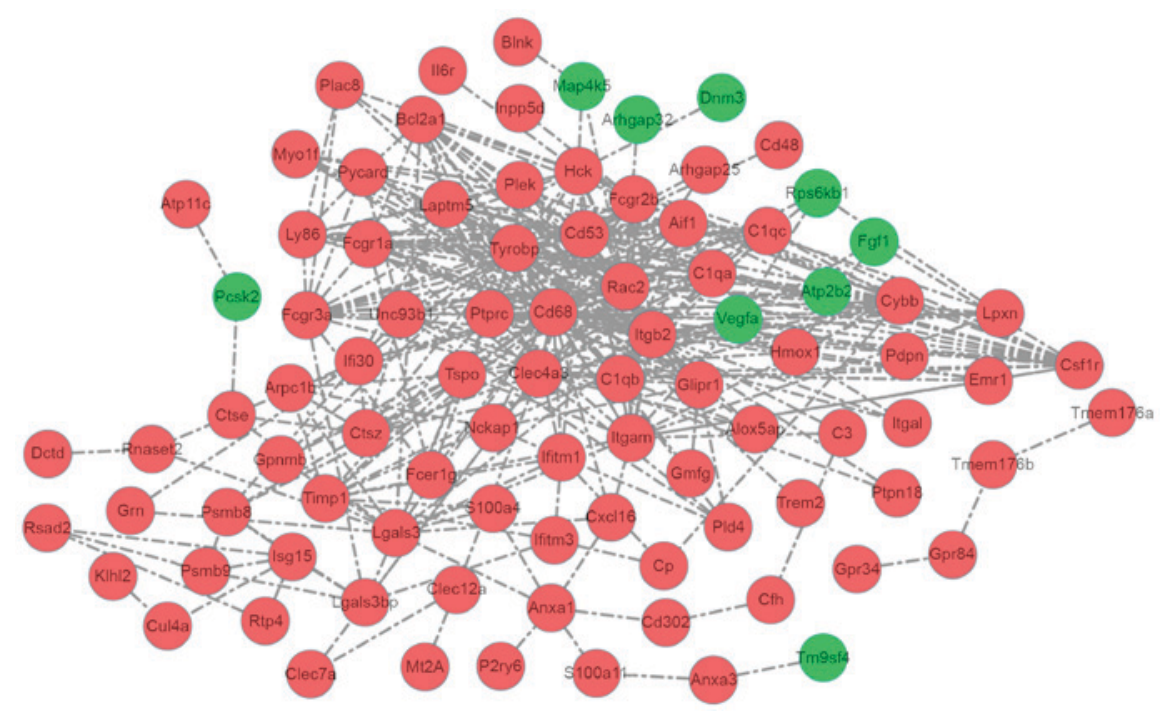

Figure 4. The protein-protein interaction network of co-expressed genes. The red nodes represent upregulated genes; and the green nodes represent downregulated genes. A dotted line between two nodes indicates that there is an interaction between these two nodes.

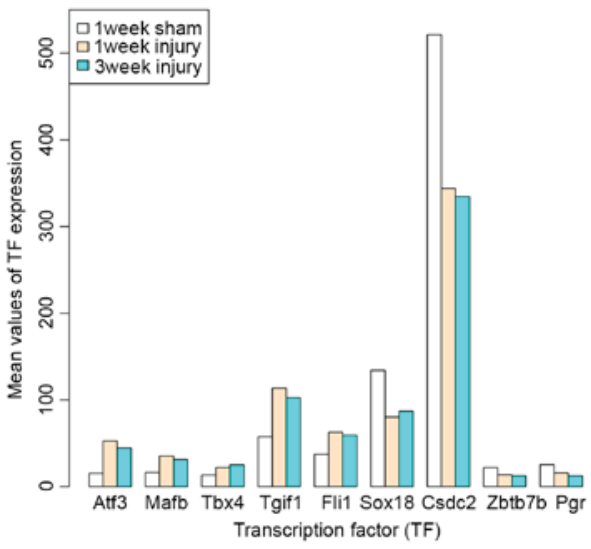

Figure 5. The bar diagram displaying the mean expression of TFs identified from the differentially expressed genes common to the 1 week and 3 week injury samples. The white bars represent lumbar spinal cord samples obtained from rats 1 week following a sham laminectomy; the yellow bars and blue bars represent lumbar spinal cord samples obtained from rats 1 and 3 weeks following contusive spinal cord injury at the T9 level. TF, transcription factor.
Tyrobp, which are associated with natural killer cell-mediated cytotoxicity, may have crucial functions in TSCI. Tgifl and $P g r$ may exert a regulatory function in TSCI. These observations require experimental validation, however they are expected to aid the elucidation of the molecular mechanisms in TSCI.

\section{References}

1. Jain NB, Ayers GD, Peterson EN, Harris MB, Morse L, O'Connor KC and Garshick E: Traumatic spinal cord injury in the United States, 1993-2012. Jama 313: 2236-2243, 2015.

2. Chen MJ, Kress B, Han X, Moll K, Peng W, Ji RR and Nedergaard M: Astrocytic CX43 hemichannels and gap junctions play a crucial role in development of chronic neuropathic pain following spinal cord injury. Glia 60: 1660-1670, 2012.

3. Chen M, Ni Y, Liu Y, Xia X, Cao J, Wang C, Mao X, Zhang W, Chen C, Chen X and Wang Y: Spatiotemporal expression of EAPP modulates neuronal apoptosis and reactive astrogliosis after spinal cord injury. J Cell Biochem 116: 1381-1390, 2015.

4. Zhu P, Hata R, Nakata K, Cao F, Samukawa K, Fujita H and Sakanaka M: Intravenous infusion of ginsenoside Rb1 ameliorates compressive spinal cord injury through upregulation of Bcl-xL and VEGF. Int J Neurol Neurother 2: 1, 2015. 
5. De Biase A, Knoblach SM, Di Giovanni S, Fan C, Molon A, Hoffman EP and Faden AI: Gene expression profiling of experimental traumatic spinal cord injury as a function of distance from impact site and injury severity. Physiol Genom 22: 368-381, 2005.

6. Byrnes KR, Garay J, Di Giovanni S, De Biase A, Knoblach SM, Hoffman EP, Movsesyan V and Faden AI: Expression of two temporally distinct microglia-related gene clusters after spinal cord injury. Glia 53: 420-433, 2006.

7. Sharp J, Frame J, Siegenthaler M, Nistor G and Keirstead HS: Human embryonic stem cell-derived oligodendrocyte progenitor cell transplants improve recovery after cervical spinal cord injury. Stem Cells 28: 152-163, 2010.

8. Shin HY, Kim H, Kwon MJ, Hwang DH, Lee K and Kim BG Molecular and cellular changes in the lumbar spinal cord following thoracic injury: Regulation by treadmill locomotor training. PLoS One 9: e88215, 2014.

9. Gautier L, Cope L, Bolstad BM and Irizarry RA: Affy-analysis of Affymetrix GeneChip data at the probe level. Bioinformatics 20: 307-315, 2004

10. Smyth GK: Limma: Linear models for microarray data. In: Bioinformatics and computational biology solutions using R and Bioconductor. Springer, pp397-420, 2005.

11. Obayashi T and Kinoshita K: Rank of correlation coefficient as a comparable measure for biological significance of gene coexpression. DNA Res 16: 249-260, 2009.

12. Kohl M, Wiese S and Warscheid B: Cytoscape: Software for visualization and analysis of biological networks. In: Data Mining in Proteomics. Springer, pp291-303, 2011.

13. Maere S, Heymans K and Kuiper M: BiNGO: A Cytoscape plugin to assess overrepresentation of gene ontology categories in biological networks. Bioinformatics 21: 3448-3449, 2005.

14. Zhang HM, Liu T, Liu CJ, Song S, Zhang X, Liu W, Jia H, Xue Y and Guo AY: Animal TFDB 2.0: A resource for expression, prediction and functional study of animal transcription factors. Nucleic Acids Res 43 (Database Issue): D76-D81, 2015.

15. Yang Y, Hwang CK, D'Souza UM, Lee SH, Junn E and Mouradian MM: Three-amino acid extension loop homeodomain proteins Meis2 and TGIF differentially regulate transcription. J Biol Chem 275: 20734-20741, 2000.

16. Huang J, Wah IY, Pooh RK and Choy KW: Molecular genetics in fetal neurology. Semin Fetal Neonatal Med 17: 341-346, 2012.

17. Kerr TC, Cuykendall TN, Luettjohann LC and Houston DW: Maternal Tgif1 regulates nodal gene expression in Xenopus. Dev Dyn 237: 2862-2873, 2008
18. Sha L, Kitchen R, Porteous D, Blackwood D, Muir W and Pickard B: SOX11 target genes: Implications for neurogenesis and neuropsychiatric illness. Acta Neuropsychiatrica 24: 16-25, 2012.

19. Ramsey SA, Klemm SL, Zak DE, Kennedy KA, Thorsson V,Li B, Gilchrist M, Gold ES, Johnson CD, Litvak V, et al: Uncovering a macrophage transcriptional program by integrating evidence from motif scanning and expression dynamics. PLoS Comput Biol 21: e1000021, 2008.

20. Didsbury J, Weber RF, Bokoch GM, Evans T and Snyderman R: Rac, a novel ras-related family of proteins that are botulinum toxin substrates. J Biol Chem 264: 16378-16382, 1989.

21. Iversen PO, Hjeltnes N, Holm B, Flatebo T, Strom-Gundersen I, Ronning W, Stanghelle J and Benestad HB: Depressed immunity and impaired proliferation of hematopoietic progenitor cells in patients with complete spinal cord injury. Blood 96: 2081-2083, 2000.

22. Numano F, Inoue A, Enomoto M, Shinomiya K, Okawa A and Okabe S: Critical involvement of Rho GTPase activity in the efficient transplantation of neural stem cells into the injured spinal cord. Mol Brain 2: 37, 2009.

23. Tian Y and Autieri MV: Cytokine expression and AIF-1-mediated activation of Rac2 in vascular smooth muscle cells: A role for Rac2 in VSMC activation. Am J Physiol Cell Physiol 292: C841-C849, 2007.

24. Hall A and Lalli G: Rho and Ras GTPases in axon growth, guidance, and branching. Cold Spring Harb Perspect Biol 2: a001818, 2010.

25. Lanier LL, Corliss BC, Wu J, Leong C and Phillips JH: Immunoreceptor DAP12 bearing a tyrosine-based activation motif is involved in activating NK cells. Nature 391: 703-707, 1998.

26. Gingras MC, Lapillonne H and Margolin JF: TREM-1, MDL-1 and DAP12 expression is associated with a mature stage of myeloid development. Mol Immunol 38: 817-824, 2002.

27. Jones KJ: Gonadal steroids as promoting factors in axonal regeneration. Brain Res Bull 30: 491-498, 1993.

28. Pakulski C: Neuroprotective properties of sex hormones. Anestezjol Intens Ter 43: 113-118, 2011 (In Polish).

29. Gonzalez SL, Labombarda F, González Deniselle MC, Guennoun R, Schumacher M and De Nicola AF: Progesterone up-regulates neuronal brain-derived neurotrophic factor expression in the injured spinal cord. Neuroscience 125: 605-614, 2004. 